\title{
Reducing Viscosity of Ultra Low Sulfur Diesel with Electric Field
}

\author{
Enpeng Du ${ }^{1, *}$, Qian Zhao ${ }^{2}$, Yongxin Xiao ${ }^{3}$, Rongjia Tao ${ }^{1}$ \\ ${ }^{1}$ Department of Physics, Temple University, Philadelphia, United States \\ ${ }^{2}$ China National Petroleum Corporation, Jilin Oil Field Company, Songyuan, P. R. China \\ ${ }^{3}$ Hongquan Middle School, Qianguoerluosi Mongolian Autonomous County, P. R. China
}

Email address:

enpeng.du@temple.edu (Enpeng Du)

${ }^{*}$ Corresponding author

\section{To cite this article:}

Enpeng Du, Qian Zhao, Yongxin Xiao, Rongjia Tao. Reducing Viscosity of Ultra Low Sulfur Diesel with Electric Field. American Journal of Aerospace Engineering. Vol. 5, No. 1, 2018, pp. 56-62. doi: 10.11648/j.ajae.20180501.18

Received: February 5, 2018; Accepted: February 25, 2018; Published: August 16, 2018

\begin{abstract}
Dr. Tao's viscosity theory is a university theory can be applied to a lot of liquid suspensions with the discrepancy in permittivity or permeability between the suspended contents and the base liquid. Diesel is a mixture and can be considered as one of these liquid suspensions. We ever got pretty good test results with diesel by the electric field treatment.. But, as we know, the "2007 Highway Rule" is taking effect for a while, that is, the allowable sulfur content for ULSD (15 ppm) is much lower than the previous U.S. on-highway standard for low sulfur diesel (LSD, $500 \mathrm{ppm}$ ). With Dr. Tao`s theory, if the effect is only from nanoscale sulfur aggregate, once the sulfur was taken off, the effect will be decreased or even disappear. But our laboratory test shows that ULSD has $28.7 \%$ flow rate increased after we successfully chosen an optimal field strength and an optimal treatment time, which is even higher than high sulfur diesel. This makes Dr. Tao`s Viscosity Theory can be widely used and proofed it is a nanoscale structure changing reduced the viscosity which can reduce pollution and improve engine efficacy, too.
\end{abstract}

Keywords: Ultra-Low Sulfur Diesel, Reduce Viscosity, Electric Field, Energy Security, Suppress Turbulence, Pollution

\section{Introduction}

\subsection{Diesel Is a Very Important Fossil Fuel}

Diesel, in general, is any liquid fuel used in diesel engines. The most common is a specific fractional distillate of petroleum fuel oil, but alternatives that are not derived from petroleum, such as biodiesel, biomass to liquid or gas to liquid diesel, are increasingly being developed and adopted. ULSD is the low sulfur diesel fuel portion of EPA's HeavyDuty Highway Diesel rule (the "2007 Highway Rule"), which was finalized in January 2001, is now taking effect for a while (2007). The allowable sulfur content for ULSD is 15 ppm, which is much lower than the previous U.S. onhighway standard for LSD (500 ppm). Diesel is a very important fossil fuel about $20 \%$ of the whole petroleum consumed in U.S. in 2009 (total petroleum consumption was 18.7 million barrels per day and $35 \%$ of all the energy we consumed). Diesel has some benefits compared to gasoline. First, diesel engines have more torque. This means that they can perform better under stress. Such as, they are pulling a heavy payload. This is why freight trucks, heavy industrial trucks and construction vehicles; Second, diesel-powered engines are more efficient because diesel fuel has a higher energy density than gasoline; Third, When it comes to service and maintenance, though simple upkeep is cheaper for gasoline engines, diesel engines are often cheaper to maintain in the long run. This is because they respond so much better to stress, causing them to wear out much more slowly than gasoline engines. In general, diesel engines can go about three times as far as gasoline engines before needing serious maintenance. 


\subsection{How does Diesel Engine Work}

The diesel engine gets its name from its inventor, German scientist Rudolf Diesel. The easiest way to understand how a diesel engine works is by understanding the phrase "suck, squeeze, bang, and blow". This refers to a cycle of 4 strokes known as the OTTO cycle. That it intakes air and compresses it, and then injects the fuel directly into the combustion chamber (direct injection). The diesel droplets combine with air, vaporize, then the heat of the compressed air that lights the fuel droplets. A Huge amount of heat is released, the pressure will push the piston to do work. Then exhaust stroke, the engine is ready for next cycle. (Figure 1)

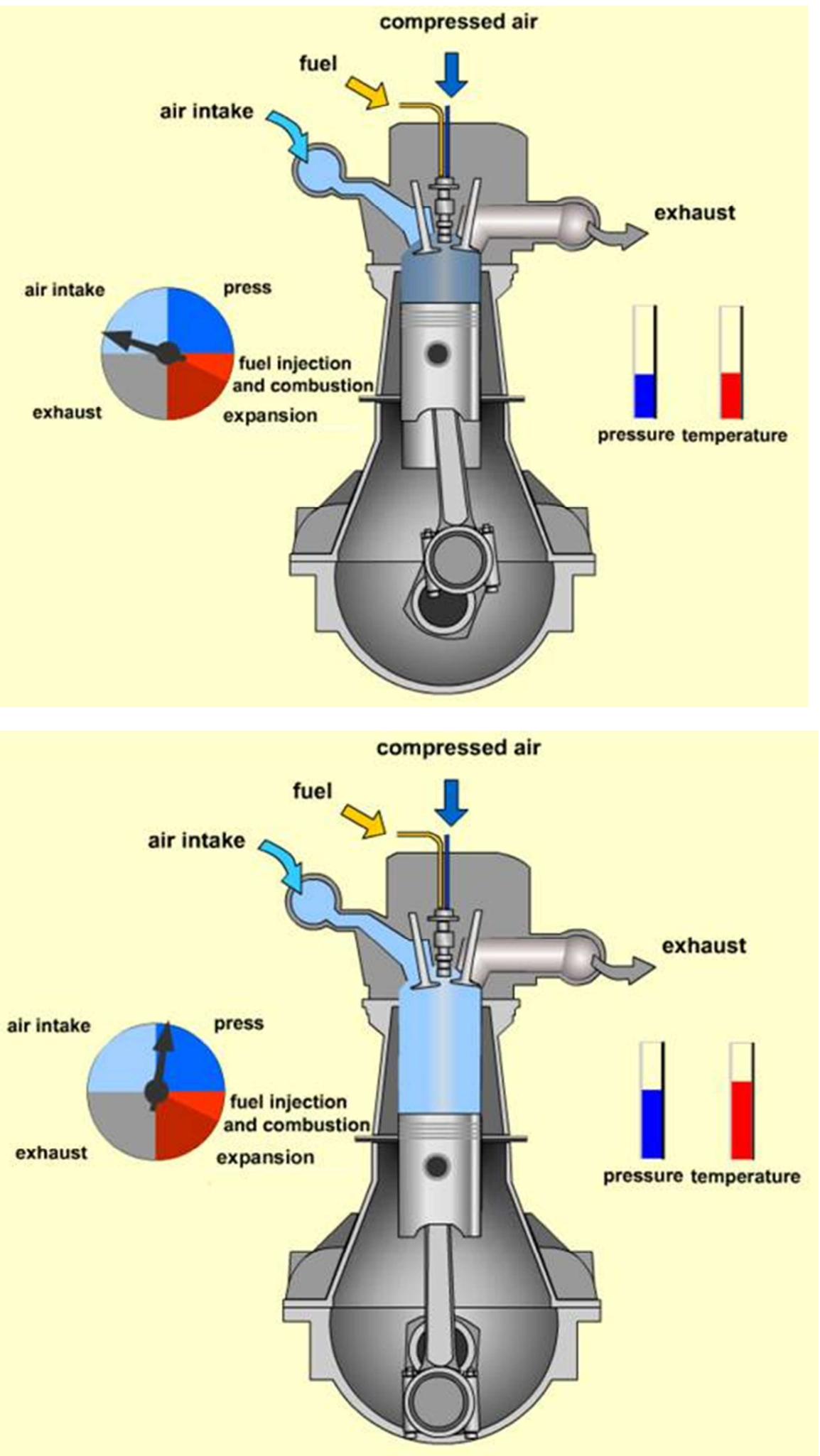




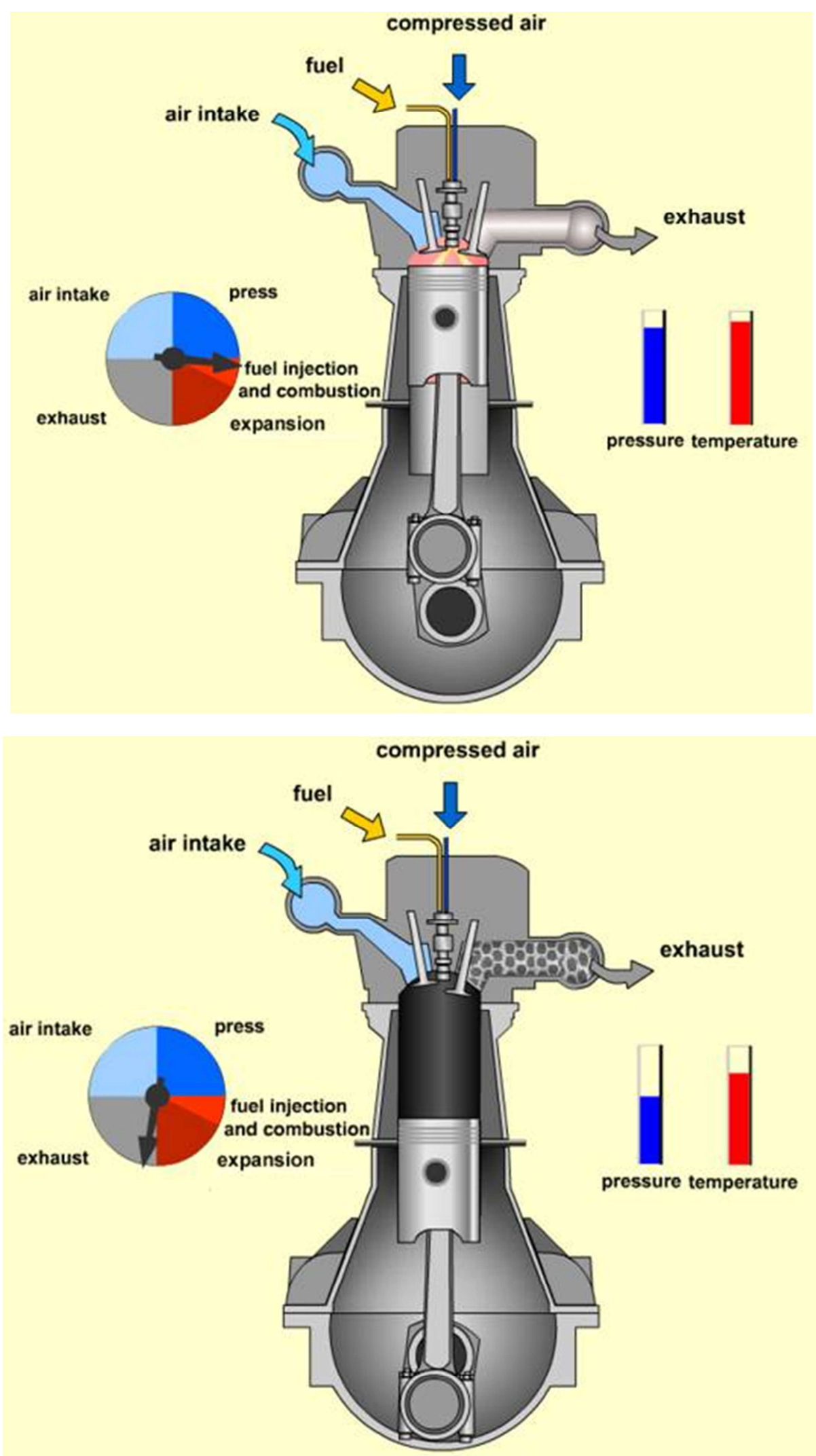

L1st: intake stroke; L2nd: compression stroke; L3rd combustion stroke; R: exhaust stroke.

Figure 1. Four-stroke Engine Cycles of An Internal Combustion Engine.

In case, the engine will do work only the process of combustion and the initial process of combustion has more efficiency. So let's take a close look at The Diesel Combustion Process. Conventional diesel combustion is 
differently from spark-ignited gasoline engines. In gasoline engines, the combustion chamber is filled with a homogeneous air-fuel mixture first, then compressed and ignited by a spark. The flame started from the spark plug then radiates away and across the combustion chamber, until the air-fuel mixture is completely burned. But the case of conventional diesel engines is a big difference, the combustion chamber is filled only with air and possibly EGR gases during the compression stroke. With high compression ratios (which are around 18:1 much higher than gasoline engines, which are somewhere around 9:1), the air is highly compressed and heats up rapidly as the piston approaches TDC (top dead center). The diesel injection system then begins injecting fuel into the superheated air and ignited by the heat of air after a number of steps.

The problem is that the air-fuel mixture is very rich in some areas and very lean in others, and some droplets are bigger, others are smaller. The fuel droplets are ignited by absorbing heat from the air. It is important to note that the diesel fuel does not ignite the moment that injection begins. During the ignition delay period, the fuel is absorbing heat as it is mixing with the turbulent air. It is desirable to have as short a delay period as possible, long delay periods mean that when the fuel does start to burn, it does so extremely fast, leading to rapid pressure rise and knock. This can also lead to an increase in NOx emissions.

The delay period depends on a number of factors some of them depends on the diesel fuel itself. A major factor which we can control is a number of small size droplets and density.

Small size droplets are easy to vaporize and ignite quickly, they will release heat to warm up the combustion chamber. Then, combustion chamber temperatures will rise to the point that it doesn't take long for the larger droplets, which is the larger amount of fuel also start burning and a rapid pressure rise takes place in the cylinder.

A short delay period allows for a smoother pressure rise during premix burning and minimizes combustion noise and emissions.

So reducing the size of fuel droplets would increase the total surface area to start burning, decrease the incomplete burning and shorter the burning time, leading to a cleaner and more efficient engine. This concept has been widely accepted because the discussions about the future engine for efficient and clean combustion are focused on ultra-dilute mixtures at extremely high pressure to produce a much finer mist of fuel for combustion. [6-10] Following this idea, the high pressure of 100 bar fuel injector can reduce the size of gasoline droplets to $25 \mu \mathrm{m}$ in diameter. And increase the pressure would require substantial changes in the fuel lines in vehicles.

Here, we present Dr. Tao`s technology for efficient combustion based on the new physics principle that proper application of electro-rheology can reduce the viscosity of petroleum fuels. $[11,12,33]$ Another way to think of it is when air molecules are packed so close together, fuel has a better chance of reacting with as many oxygen molecules as possible.

\section{Dr. Tao`s Viscosity Theory}

Crude oil is a mixture of many different molecules. Gasoline, kerosene, and diesel, the liquid made of small hydrocarbon molecules, have very low viscosity. If we treat the rest large molecules, paraffin particles, and asphalt particles etc. as suspended particles in such low viscosity base liquid made of gasoline, kerosene, and diesel, crude oil is a liquid suspension. These suspended particles are typical of the nanoscale. The theory about liquid suspensions thus provides the physics basis for our new method to reduce the viscosity of crude oil.

Einstein first studied a dilute liquid suspension of noninteracting uniform spheres in a base liquid of viscosity $\eta_{0}$ and found the effective viscosity $\eta$ as follows [13-15],

$$
\eta=\eta_{0}(1+2.5 \phi)
$$

Where the small parameter $\eta_{0}$ is the volume fraction of the suspended particles.

Following Einstein's work, Krieger-Dougherty introduced the intrinsic viscosity $[\eta]$ for particles of different shapes and generalized it for all volume fractions [16],

$$
\eta / \eta_{0}=\left(1-\phi / \phi_{m}\right)^{-[\eta] \phi_{m}}
$$

Where $\phi_{m}$ is the maximum value fraction allowed for packing the suspended particles. When $\phi$ is unchanged, the most widely used method to reduce viscosity $\eta$ is to reduce $\eta_{0}$, such as raising the temperature. On the other hand, Eq. (2) suggests that there is another method: if we change the rheology of the suspension to increase the value of $\phi_{m}$ and lower intrinsic viscosity $[\eta]$, we will reduce the viscosity $\eta$. The physics is clear: the effective viscosity depends on how much freedom the suspended particles have in the suspension. A high $\phi_{m}$ and low $[\eta]$ mean high freedom for the suspended particles, which leads to lower dissipation of energy and lower viscosity [17].

The following three mechanisms contribute to the viscosity reduction [17-18]:

(1) Aggregate the nanoscale particles into short chains with their shapes streamlined along the flow direction; (2) Increase the polydispersity to increase $\phi_{m}$; (3) Increase the average size of suspended particles.

Our technology is illustrated in Figure 2. The crude oil flows from left to right along a pipe. Initially, the nanoscale particles are randomly distributed and the viscosity is high. When the oil passes a strong local electric field, the suspended particles are polarized by the electric field. The induced dipolar interaction forces the nanoscale particles to aggregate into micrometer-size short chains. They have high polydispersity and large size. In addition, the most important are that they are of streamline shape with low $[\eta]$ along the flow direction as the electric field is parallel to the flow direction.

It is also important to note that after formation of short- 
chains along the field direction, similar to the flow of nematic liquid crystal with its molecular alignment parallel to the flow direction which breaks the rotational symmetry, making the viscosity of crude oil anisotropic. Along the field direction, the viscosity is significantly reduced, while the viscosity along the directions perpendicular to the field is actually increased [19].

This fact is very important and very useful as it does not only improve the flow along the field direction but suppresses the turbulence inside the pipeline.

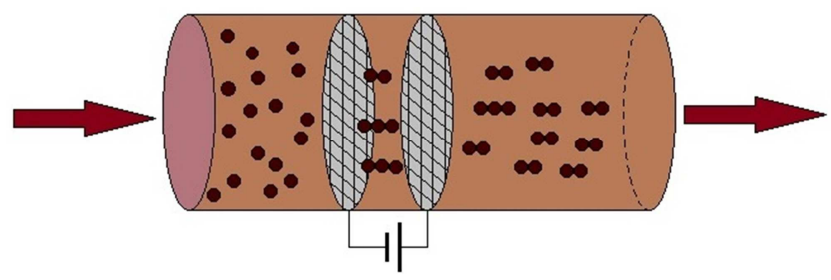

Figure 2. As the crude oil flow passes a strong local electric field, the suspended particles aggregate along the field direction, and the viscosity along the flow direction is reduced.

\section{Application of Dr. Tao's Viscosity Theory to Reducing Viscosity}

It is clear from the above background that aggregating the nanoscale particles into short chains with their shapes streamlined along the flow direction will reduce the effective viscosity while $\phi$ remains the same. At the same time, it is important to note that after formation of short-chains along the field direction, its molecular alignment parallel to the flow direction which breaks the rotational symmetry, making the viscosity of crude oil anisotropic. Along the field direction, the viscosity is significantly reduced, while the viscosity along the directions perpendicular to the field is actually increased. For most suspensions, this aggregation can be realized with either electric or magnetic fields. We assume that the particles have an electric dielectric constant $\varepsilon_{p}$ different from the dielectric constant of the base liquid $\varepsilon_{f}$. In an electric field, the particles are thus polarized along the field direction. The dipole moment is estimated by, $\vec{p}=\vec{E} a^{3} \varepsilon_{f}\left(\varepsilon_{p}-\varepsilon_{f}\right) /\left(\varepsilon_{p}+2 \varepsilon_{f}\right)$ where $\vec{E}$ is the local electric field, which should be close to the external field in dilute cases. The dipolar interaction between the two induces electric dipoles is $U=p^{2}\left(1-3 \cos ^{2} \theta\right) /\left(\varepsilon_{f} r^{3}\right)$, where $\mathrm{r}$ is the distance between these two dipoles and $\theta$ is the angle between the joining line and the electric field. If this interaction is stronger than the thermal Brownian motion, that is $\alpha=p^{2} n /\left(\varepsilon_{f} k_{B} T\right) \geq 1$, these two dipoles will aggregate together to align in the field direction. $\alpha=p^{2} n /\left(\varepsilon_{f} k_{B} T\right) \geq 1$, where $\mathrm{n}$ is the particle number density, $k_{B}$ is the Boltzmann constant and $T$ is the absolute temperature. We derive the following critical field

$$
E_{c}=\left[k_{B} T /\left(n \varepsilon_{f}\right)\right]^{1 / 2}\left(\varepsilon_{p}+2 \varepsilon_{f}\right) /\left[a^{3}\left(\varepsilon_{p}-\varepsilon_{f}\right)\right]
$$

If the applied electric field is weaker than $E_{c}$, the thermal Brownian motion prevents particles from aggregating together. So the applied electric field must be not lower than $E_{c}$.

The required pulse duration time is,

$$
\tau=n^{-1 / 3} / v=\pi \eta_{0}\left(\varepsilon_{p}+2 \varepsilon_{f}\right)^{2} /\left[\varepsilon_{f} n^{5 / 3} a^{5}\left(\varepsilon_{p}-\varepsilon_{f}\right)^{2} E^{2}\right]
$$

If the duration of the electric field is too shorter than $\tau$, the particles do not have enough time to aggregate together. [4]

Once the e field is turned off, the hysteresis time is, $t=\left(3 a^{3} \pi \eta_{0}\right) /\left(K_{B} T\right)$.

Here, we present Dr. Tao`s technology for efficient combustion based on the new physics principle that proper application of electro-rheology can reduce the viscosity of petroleum fuels which has been verified by the nuclear method and can be simulated, too. [1, 20-29, 32] Another way to think of it is when air molecules are packed so close together, fuel has a better chance of reacting with as many oxygen molecules as possible.

Reducing the fuel viscosity improves the fuel atomization. The influence of fuel's viscosity on the atomization can be illustrated by the equation 1 . The average droplet size can be estimated as

$$
\mathrm{D}=\eta^{2} / \rho \sigma O h_{c}
$$

Where D is the droplet's diameter and $\eta, \rho$, and $\sigma$ are the fuel's viscosity, density, and surface tension, the universal critical value $\mathrm{Oh}_{\mathrm{c}}$ close to 0.1 for the Ohnesorge number, respectively. As the viscosity $\eta$ is reduced, the average droplet size is reduced dramatically. The result from Spray experiment indicates that electric field is very effective on improve performance on diesel automation. This result also suggests that diesel engines would benefit from our device by improving fuel efficiency and reducing emission pollution.

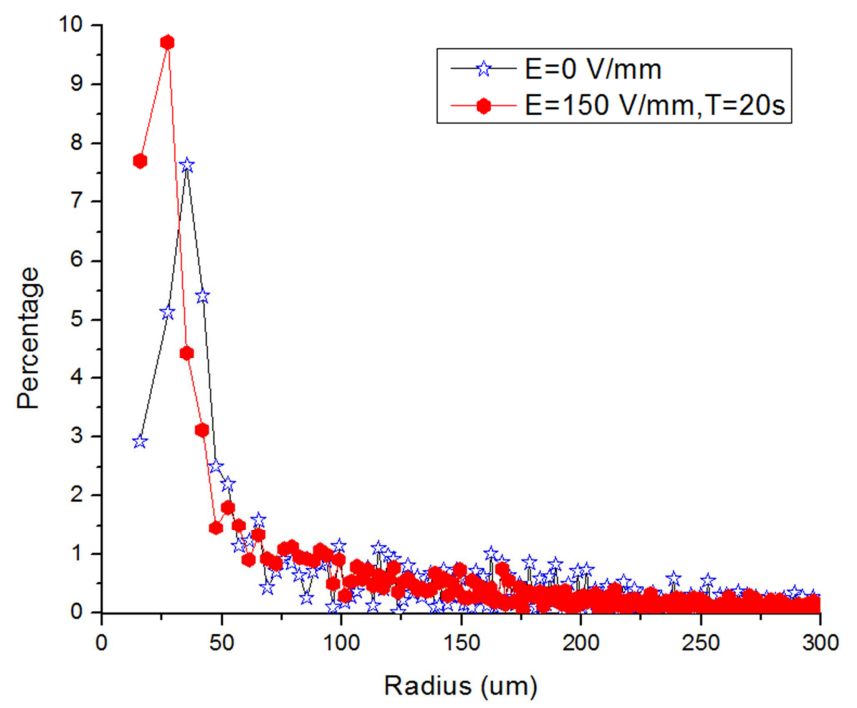

Figure 3. The droplet size from the diesel sprays with field treatment of 1500 $\mathrm{V} / \mathrm{mm}$ for $20 \mathrm{~s}$ and without Electric field. 


\section{The ULSD Experiments}

We made a device (Figure 2 and Figure 4) for the laboratory test. Inside the container, there are three metallic meshes, serving as three electrodes. A high voltage can be applied on the middle mesh and both the upper and lower are grounded, so three meshes produced an electric field in the flow direction. At the bottom of the container, there is a $50 \mathrm{~mm}$ long capillary with wide open edge in order to measure the flow rate and reduce the surface extension.

During the test, the device is placed vertically. We fill the container with ultra low surfer diesel sample to cover the upper electrode mesh and measure the flow rate with digital balance controlled by Lab View. Then we will apply electric field treatment to the ultra low surfer diesel sample to reduce the viscosity. We refill the container with the exact same volume diesel and apply an electric field for a selected time interval. Then we measure the flow rate of the treated diesel sample.

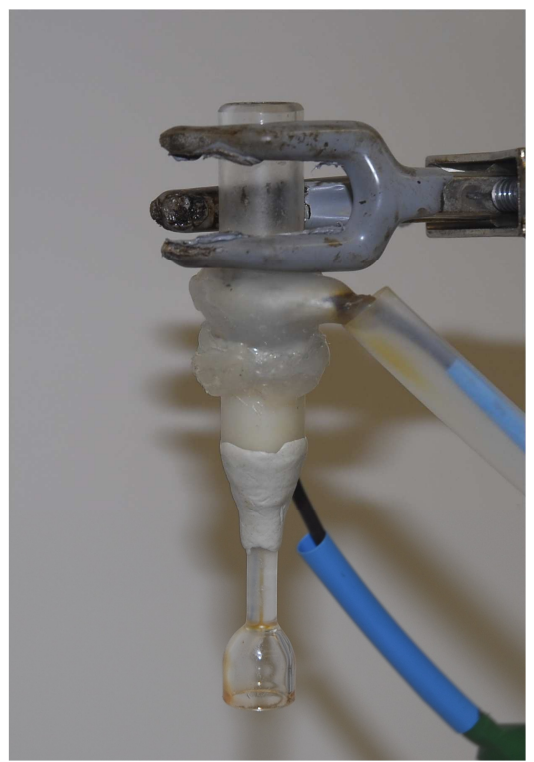

Figure 4. Three-mesh capacitor electric field treatment instrument.

\section{Results}

With this method, we found that the required electric field is much stronger compared with high sulfur diesel (bunker diesel) and low sulfur diesel. Table 1. [3, 4, 11, 12]

Table 1. Electric field treatment for different diesel samples which has different sulfur level.

\begin{tabular}{lllll}
\hline & E & T (second) & Increase & Duration \\
\hline HSD & $500 \mathrm{v} / \mathrm{mm}$ & 2 & $30 \%$ & 2 hours \\
LSD & $1000 \mathrm{~V} / \mathrm{mm}$ & 2 & $30 \%$ & 60 minutes \\
ULSD & $2500 \mathrm{~V} / \mathrm{mm}$ & 10 & $20 \%$ & 10 minutes \\
\hline
\end{tabular}

For theULSD, we have to apply much high electric field. As shown in Figure 5, when we apply an electric field of $2500 \mathrm{v} / \mathrm{mm}$ for $10 \mathrm{~s}$, the flow rate increased about $20 \%$, indicating that the viscosity of treated diesel is reduced more than $20 \%$. However, this viscosity reduction does not last very long. In about 10 minutes, the flow rate of treated diesel and untreated diesel become almost the same, indicating that the viscosity returns to the original value. For diesel with high sulfur and low sulfur level diesel, the viscosity reductions can last for more than hours.

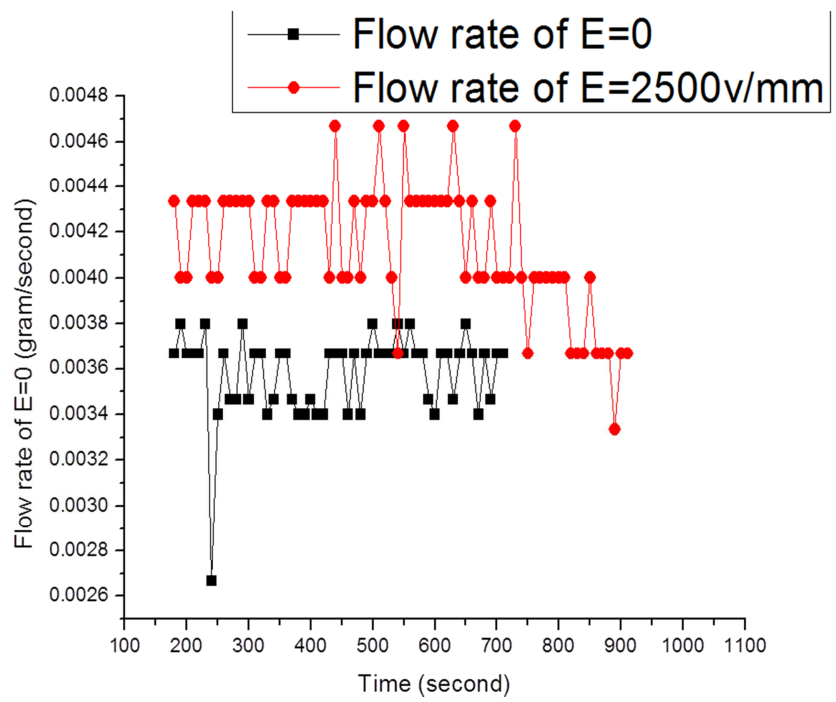

Figure 5. At E=2500V/mm for 10 seconds, the ULSD sample viscosity is reduced by $20 \%$, and it is only last hundred of seconds.

\section{Conclusion}

This phenomenon is easy to understand. In ULSD, the electric field forces the large molecules to aggregate. Because the large molecules are much smaller than nanoscale sulfur particles of high sulfur diesel and low sulfur diesel, the required electric field is much stronger. Also because the large molecules are much lighter than the sulfur particles, they are much easier to be kicked out from aggregated chains by the thermal vibrations. This kind of nanoscale molecules aggregation is verified by Small Angle Neutron Scattering experiment at NIST. [5] And with mew technology, we can make the electric field even stronger. $[30,31]$

So with Dr. Tao`s viscosity reduce method, we can reduce the droplets size and increased fuel efficiency, minimized emissions and improved startup and acceleration, run smoother and stronger over its service life. In the situation of emission control standards continue to tighten, this and other ideas will be implemented as engine manufacturers work to extend the life of the internal combustion engine.

\section{References}

[1] $\mathrm{R}$ Tao, $\mathrm{H}$ Tang, $\mathrm{K}$ Tawhid-Al-Islam, E Du, J Kim, "Electrorheology leads to healthier and tastier chocolate" Proceedings of the National Academy of Sciences 113 (27), 7399-7402 (2016).

[2] K Tawhid-Al-Islam, R Tao, E Du, H Tang, X Xu, M Autieri, "Suppressing Turbulence and Reducing Blood Viscosity to Prevent Heart Attacks and Strokes" Bulletin of the American Physical Society (2017). 
[3] E. Du, L. Cai, K. Huang, H. Tang, X. Xu and R. Tao," Reducing Viscosity to Promote Biodiesel for Energy Security and Improve Combustion Efficiency ",

DOI:10.1016/j.fuel.2017.9.055 211C pp. 194-196 Fuel, (2018).

[4] E. Du, X. Xu, K. Huang, H. Tang and R. Tao, "Bunker Diesel Viscosity is Drmatically Reduced by Electrorheological Treatment", International Journal of Modern Physics B, 1850012, Volume: 31, (2017).

[5] Rongjia Tao, Enpeng Du, Hong Tang, "Neutron Scattering Studies of Crude Oil Viscosity Reduction with Electric Field," Fuel, Volume 134, 493-498, (2014).

[6] Aoyagi, Y.; et al, SAE Tech. Pap. 2006-01-0077 (2006).

[7] Hiroshi, M.; et al. FISTA World Automotive Congress, Barcelona, Spain, May 23-27, 2004.

[8] Pirault, J. A.; Klippenstein, S. J. J. Phys. Chem. A 2006, 110, 10528-10544.

[9] John B. Heywood, Internal Combustion Engine Fundamentals (1988).

[10] A. H, Lefebvre, Atomization and Sprays, (Taylor \& Francis, 1989) 27-73.

[11] E. Du, H. Tang, K. Huang, and R. Tao, "Reducing the viscosity of diesel fuel with electrorheological effect," Journal of Intelligent Material Systems and Structures, V22, 17131716 (2011).

[12] E. Du, L. Cai, Q. Zhao and R. Tao, " Electric Field Suppressed turbulence and Reduced Viscosity of Cenovus Crude Oil Sample ", Fuel, (2018).

[13] Einstein A. On the movement of small particles suspended in stationary liquids required by the molecular-kinetic theory of heat. Ann Physik 1905; 17:549-60.

[14] Einstein A. A new determination of molecular dimensions. A Ann Physik 1906; 19:289-306.

[15] Einstein A. On the theory of brownian motion. Ann Physik $1906 ; 19: 371-81$.

[16] Krieger IM, Dougherty TJ. A mechanism for non-newtonian flow in suspensions of rigid spheres. Trans Soc Rheol 1959; $3: 137-52$.

[17] E. Du, Q. zhao, Y. Xiao, L. Cai, and R. Tao, "Electric field suppressed turbulence and reduced viscosity of asphaltene base crude oil sample" Volume 220, 15 May 2018, Pages 358362.

[18] K. Huang, E. Du, H. Tang, and R. Tao, "Electrorheology improves E-85 engine efficiency and performance," Journal of Intelligent Material Systems and Structures, V 22, 1707-1711 (2011).

[19] Miesowicz M. The three coefficients of viscosity of anisotropic liquids. Nature 1946; 158:27.

[20] Du, Enpeng Neutron scattering studies of crude oil viscosity reduction with electric field; AAT 3719351; ISBN: 9781321996807; Dissertation Abstracts International, Volume: 76-12 (E), Section: B (2015).
[21] Zhang, Wen Ling, et al. "Growth of Polyaniline Nanoneedles on MoS2 Nanosheets, Tunable Electroresponse, and Electromagnetic Wave Attenuation Analysis." The Journal of Physical Chemistry C 121.9 (2017): 4989-4998.

[22] Du, Enpeng, et al., "Study of quadrupole interactions in nano$\mathrm{LaFeO} 3$ perovskite by time differential perturbed angular correlation", Nuclear Techniques, Vol. 28, No. 6, P. 409-412 (2005).

[23] Zhu, SY; Iwata, T; Xu, YJ; Zheng, YN; Zhou, DM; Zhu, JH; Wang, ZQ; Yuan, DQ; Du, Enpeng; Zuo, Y,'Experimental verification of heavy ion irradiation simulation", MODERN PHYSICS LETTERS B, Volume: 18, Issue: 17, pp. 881-885 (2004).

[24] Zhou, DM; Zheng, YN; Zhu, JZ; Xu, YJ; Du, Enpeng; Wang, ZQ; Yuan, DQ; Luo, HL; Rong, CF; Mihara, M; Fukuda, M; Matsuta, K; Minamisono, T; Zhu, SY, "beta-NMR and betaNQR spectrometer", HIGH ENERGY PHYSICS AND NUCLEAR PHYSICS-CHINESE EDITION Volume: 28 Issue: 3 Pages: 294-298 (2004).

[25] Zhou, DM; Zheng, YN; Zhu, JZ; Xu, YJ; Du, Enpeng; Wang, ZQ; Luo, HL; Yuan, DQ; Rong, CF; Mihara, M; Fukuda, M; Matsuta, K; Minamisono, T; Zhu, SY, "Development of betaNMR and beta-NQR", CHINESE PHYSICS LETTERS Volume: 20 Issue: 10 Pages: 1698-1701 (2003).

[26] $\mathrm{Wu}$, Fengfeng, et al. "Electrofluid hydrolysis enhances the production of fermentable sugars from corncob via in/reversephase induced voltage." Bioresource Technology 234 (2017): 158-166.

[27] Rongjia Tao, Enpeng Du, Hong Tang, "Neutron Scattering Studies of Crude Oil Viscosity Reduction with Electric Field," Fuel, Volume 134, 493-498, (2014).

[28] Chenbo Ma, Yingda Lu, Chaohui Chen, Kai Feng, Zixin Li, Xinyi Wang, and Jinjun Zhang "Electrical Treatment of Waxy Crude Oil To Improve Its Cold Flowability" Ind. Eng. Chem. Res., $56 \quad(38), \quad$ pp $10920-10928$ DOI: 10.1021/acs.iecr.7b02140 (2017).

[29] Tada, Shigeru, Yan Shen, and Zhiyong Qiu. "Modeling and simulation of dielectrophoretic collective dynamics in a suspension of polarizable particles under the action of a gradient AC electric field." Electrophoresis (2017).

[30] Tan, T. et al. Magnesium diboride coated bulk niobium: a new approach to higher acceleration gradient. Sci. Rep. 6, 35879; doi: 10.1038/srep35879 (2016).

[31] C Zhuang, T Tan, A Krick, Q Lei, K Chen, XX Xi "MgB2 thin films on metal substrates for superconducting RF cavity applications" Journal of superconductivity and novel magnetism 26 (5), 1563-1568.

[32] Raheek I. Ibrahim, Manal K. Oudah, Aws F. Hassan, "Viscosity reduction for flowability enhancement in Iraqi crude oil pipelines using novel capacitor and locally prepared nanosilica", Journal of Petroleum Science and Engineering 156 (2017) 356-365.

[33] E. Du, Q Zhao, Y Xiao, L Cai, R Tao, "Electric field suppressed turbulence and reduced viscosity of asphaltene base crude oil sample", Fuel 220, 358-362 (2018). 